\title{
Scénariser l'apprentissage, une activité de modélisation
}

Richard Hotte

Centre de recherche LICEF-TÉLUQ/UQAM, CANADA

Richard.Hotte@licef.teluq.uqam.ca

\section{Hélène Godinet}

Équipe EducTice, Institut national de recherche pédagogique, Lyon, FRANCE

Helene.Godinet@inrp.fr

Jean-Philippe Pernin

Équipe EducTice, Institut national de recherche pédagogique, Lyon, FRANCE Jean-Philippe.Pernin@inrp.fr

\section{Introduction}

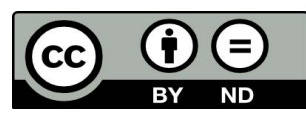

(C)Auteur(s). Cette œuvre, disponible à http://www.ritpu.org/IMG/pdf/ritpu0402_hotte.pdf, est mise à disposition selon les termes de la licence Creative Commons Attribution - Pas de Modification 2.5 Canada : http://creativecommons.org/licences/by-nd/2.5/ca/deed.fr

\section{Un regard sur la scénarisation pédagogique}

Depuis le début des années 2000, un ensemble de travaux de recherche et d'initiatives de terrain visent à proposer ou à utiliser des modèles, des méthodes et des outils pour concevoir, mettre en place, exploiter et analyser les scénarios d'apprentissage. Sur le plan international, on peut citer en particulier les travaux menés par Koper et Tattersall, (2005) portant sur les langages de modélisation pédagogique et plus spécifiquement autour de la proposition IMS (Instructional Management System) Learning Design ainsi que ceux de Paquette (2006) conduisant à développer une approche en ingénierie éducative supportant le développement d'environnements d'apprentissage en ligne. Ces travaux ont contribué à faire émerger, au sein des communautés éducatives, le concept de scénario pédagogique comme un composant essentiel des systèmes d'apprentissage en ligne. Le but poursuivi par ces chercheurs et leurs équipes est « d'explorer comment améliorer la qualité pédagogique des cours en ligne, dans une voie interopérable, avec les outils d'usage facile ».

Dans cette perspective, le scénario pédagogique se définit comme l'orchestration d'un ensemble d'activités d'apprentissage auxquelles s'ajoutent, d'une part, la description des ressources utiles à leur réalisation et, d'autre part, les productions de l'apprenant qui en découlent. La modélisation pédagogique - c'est-à-dire l'acte pédagogique par lequel les différentes activités, ressources et services pédagogiques sont agrégés de façon à offrir une solution de formation - est au cœur de la conception des processus d'apprentissage intégrant des technologies numériques. En fait, elle concerne aussi bien la description statique des scénarios pédagogiques que la description de leur fonctionnement dynamique lors de leur déploiement dans un environnement informatique.

Modéliser et opérationnaliser un scénario, dans ses dimensions statique et dynamique, sont des tâches complexes. Cette complexité concerne aussi bien l'enseignant qui planifie les activités de l'apprenant que l'informaticien responsable du développement d'outils supports à ces activités. La modélisation d'un scénario est donc le résultat d'une intervention multi-acteurs qui doit être supportée par des méthodes, des techniques de conception (design) et des outils pour assurer la qualité pédagogique, et par des spécifications (comme l'IMS LD) pour permettre la réutilisation et l'interopérabilité au sein de banques d'objets d'apprentissage. Le scénario représente alors un processus d'apprentissage qui se décline en un ou plusieurs cheminements possibles pour l'apprenant ou encore qui offre une collection d'activités et de ressources pédagogiques faiblement couplées. C'est alors aux acteurs de construire de façon dynamique le processus de parcours de ces activités. 
La scénarisation de situations d'apprentissage est une activité d'ingénierie qui s'inscrit au cœur de la pratique de l'enseignant ou du formateur engagé dans la conception de dispositifs de formation, en présence, à distance et en ligne. Nous assistons aujourd'hui à un accroissement constant des initiatives visant à compléter (voire à substituer) les modalités de formation existantes par la mise à disposition de ressources numériques ou de moyens de communication informatiques. Les situations d'apprentissage visées peuvent concerner aussi bien l'enseignement fondamental que les dispositifs de formation professionnelle; elles s'appuient sur des modalités de plus en plus variées, mêlant apprentissage individuel ou collaboratif, activités en présence ou à distance et travail synchrone ou asynchrone. «Il s'agit notamment pour un enseignant de trouver ou de créer les ressources les mieux adaptées à ses objectifs, de les rendre aisément disponibles aux élèves, de les intégrer et de les orchestrer au sein de scénarios pertinents » (Pernin et Godinet, 2006).

Comme le rapporte Savard (2007) dans le compte-rendu du colloque Scénario 2007, les tenants de la scénarisation formalisée et de la planification détaillée diront que l'enseignement et l'apprentissage sont des activités si importantes et si fragiles qu'elles doivent être planifiées dans les moindres détails pour assurer un maximum d'efficacité. Ceux qui se montrent plutôt agacés par cette formalisation objecteront qu'il est impossible de planifier les actions et réactions d'un être humain et encore moins celles d'un groupe d'êtres humains. Ils diront aussi que la pédagogie est souvent le théâtre d'une douce euphorie qui ne se planifie pas, mais qui fait que le message ou, mieux encore, les passions passent et rendent les apprentissages merveilleux. Tous ont pourtant pour même but ultime de faciliter l'apprentissage.

L'arrimage entre les travaux et les développements dans les domaines de l'ingénierie éducative et du learning design et la pratique de l'enseignement ne va pas nécessairement de soi. Pour tenter d'interroger cette problématique de la scénarisation pédagogique, de se l'approprier et de la discuter selon un éclairage interdisciplinaire, deux colloques internationaux se sont tenus successivement. L'Institut national de recherche pédagogique (INRP) de France a pris l'initiative du premier sous le thème Scénariser l'enseignement et l'apprentissage : une nouvelle compétence pour le praticien? Le deuxième a été pris en charge par le Centre interuniversitaire de recherche en technologies d'apprentissage (CIRTA) du Québec sous le thème Scénariser les activités de l'apprenant: une activité de modélisation et en collaboration avec des chercheurs de l'INRP à Lyon.

\section{Les colloques internationaux Scénario 2006 et Scénario 2007}

\section{Lyon, avril 2006. Scénariser l'enseignement et l'apprentissage : une nouvelle compétence pour le praticien?}

Désireux d'explorer la problématique de l'étude et de l'accompagnement de la mutation progressive des métiers de l'éducation et de la formation, l'INRP de France a organisé la première édition d'un événement scientifique international spécifiquement consacré à la thématique de la scénarisation pédagogique en avril 2006 dans le cadre de la Biennale de l'éducation. Ce premier colloque, Scénario 2006, avait pour thématique Scénariser l'enseignement et l'apprentissage : une nouvelle compétence pour le praticien? Il a permis de réunir près de 70 chercheurs ou praticiens francophones de différentes disciplines et de confronter leurs points de vue scientifiques et leurs expériences. Il a donné lieu à l'édition d'actes électroniques consultables en ligne à l'adresse : http:/ / www.inrp.fr/publications / edition-electronique/. Cette participation importante illustre la pertinence de la thématique, à un moment où l'usage des technologies numériques se généralise dans la sphère privée et où, chaque jour davantage, des ressources et des outils informatiques sont mis à la disposition des acteurs éducatifs (enseignants, formateurs, apprenants, etc.).

Ce premier colloque visait en particulier à dresser un inventaire des questions de recherche et des pratiques de terrain gravitant autour des termes de «scénario » et de « scénarisation ». Un premier constat concerne la diversité des acceptions affectées au terme scénario. Le recours à des expressions telles que scénario d'évaluation, scénario d'encadrement, scénario 
collaboratif, scénario d'activité sur simulateur, scénario d'usage des artéfacts ou encore scénarisation rapide d'applications multimédia affine et nuance les significations qu'on attribue au concept de scénario, et plus encore à celui de scénario pédagogique. Dès lors, la scénarisation apparaît comme une activité complexe ne concernant pas la seule planification des activités de l'apprenant. Il reste encore aujourd'hui à faire un travail important de recensement et de classification de ces variations terminologiques et sémantiques afin de disposer de glossaires partagés.

Une seconde remarque porte sur la possibilité d'étendre vers d'autres contextes la démarche de scénarisation développée à l'origine dans le cadre des dispositifs de formation à distance. Ainsi, les formalismes proposés semblent peu adéquats pour rendre compte de la complexité des situations de classe, en particulier pour traduire l'importance des aspects dynamiques et des savoir-faire existants des enseignants. On insiste sur l'importance de la dimension didactique et la nécessité de prendre en compte le partage de formalismes communs au sein de communautés d'enseignants ou de formateurs. Cette approche est appliquée particulièrement aux domaines des mathématiques, des sciences de la vie et de la Terre et de la formation d'adultes.

Le thème des modèles et des outils de conception de scénarios est fréquemment abordé. On est préoccupé de fournir des modèles permettant de pallier les carences de la proposition IMS Learning Design ou de proposer d'autres options. Certains mettent notamment l'accent sur la faiblesse de la prise en compte des aspects didactiques ou des interactions entre acteurs des situations d'apprentissage. D'autres proposent de raffiner le concept dans le cas d'activités spécifiques reposant par exemple sur l'utilisation de logiciels de simulation.

L'activité de conception de scénarios étant souvent décrite comme complexe, il paraît nécessaire de fournir aux enseignants des environnements d'assistance. On retrouve diverses démarches dont celles qui proposent d'analyser les formes de travail pour pouvoir réfléchir à la construction d'outils d'aide, d'utiliser des canevas pour illustrer les différents types de compétences ou encore de définir et de développer des environnements logiciels permettant d'assister les enseignants dans leur activité de conception de scénarios. On va même jusqu'à proposer une chaîne d'outils complémentaires couvrant les phases de conception et de mise en œuvre dans le cadre de la formation ouverte et à distance au niveau ingénieur.

Les relations entre ressources et activités ont été étudiées par plusieurs auteurs. C'est le cas de travaux fondés sur la théorie de la genèse instrumentale qui analysent l'évolution des ressources et de leurs scénarios d'usage dans le domaine précis des mathématiques. Certains proposent ainsi d'adjoindre aux ressources utilisées des « fiches » qui décrivent cette évolution selon différents points de vue.

L'évaluation des usages de scénarios a été également abordée lors de ce colloque 2006. Des travaux fournissent les premiers résultats d'une analyse de banque de scénarios au primaire visant à mesurer l'écart entre la prescription et les usages effectifs ou encore ceux d'une comparaison entre deux formes d'encadrement, proactif et réactif, dans une formation à distance universitaire.

Comme le soulignent Pernin et Godinet (2006), ce premier colloque a démontré toute la richesse d'une thématique émergente, située à la charnière de plusieurs disciplines. À la suite de cette manifestation, le comité scientifique a décidé, d'une part, de publier les actes sous un format numérique et, d'autre part, d'organiser une seconde édition en mai 2007 à Montréal.

\section{Montréal, mai 2007. Scénariser les activités de l'apprenant : une activité de modélisation}

Le colloque Scénario 2007 s'est tenu à Montréal dans les locaux de la Maison des technologies de formation et d'apprentissage (MATI) sous l'intitulé Scénariser les activités de l'apprenant : une activité de modélisation. Son contenu portait sur les activités de scénarisation pédagogique et plus spécifiquement sur la formalisation de ce type d'activités. 
Les deux conférenciers, Gilbert Paquette, du Centre de recherche LICEF de Montréal, et Pierre Dillenbourg, de l'École Polytechnique de Lausanne, ont exploré deux aspects de la scénarisation pédagogique et tenté, à leur manière, de déterminer si une scénarisation formalisée, c'està-dire interprétable par la machine, peut laisser une place à la spontanéité et conduire à un apprentissage significatif. Selon Gilbert Paquette, la problématique de l'instrumentation cognitive de la scénarisation pédagogique amène à définir la structure des activités d'apprentissage comme un processus multi-acteurs centré sur l'apprenant. Il existe différentes façons de représenter un scénario pédagogique et divers outils pour l'édition de scénarios multi-acteurs. Pour sa part, Pierre Dillenbourg aborde les méthodes de modélisation de scripts d'apprentissage collaboratif. Et cet apprentissage collaboratif n'est pas toujours efficace, car il dépend de l'intensité des interactions verbales entre étudiants pendant la collaboration. Selon Dillenbourg, « les résultats de l'apprentissage sont en particulier liés à l'émergence d'explications élaborées, à la négociation des significations, à la qualité de l'argumentation ou à la régulation réciproque des processus cognitifs ». Planifier un script revient à perturber un système « naturel » de collaboration de façon à ce que ce soit les interactions nécessaires pour maintenir la collaboration (malgré cette perturbation) qui provoquent les résultats d'apprentissage escomptés.

Deux conférences thématiques sont venues enrichir la réflexion sur des aspects particuliers traités par les conférenciers. Une première, présentée par Monique Grandbastien, professeure en informatique à l'Université Henri Poincaré de Nancy (France) et chercheure dans le domaine des environnements informatiques pour l'apprentissage humain (EIAH), a fait le point sur les travaux touchant les ontologies. Les ontologies sont des modèles de connaissances développés en ingénierie des connaissances, utilisables par des agents humains et logiciels, et indispensables dans les applications du web sémantique. Il serait souhaitable que des ontologies générales pour l'éducation existent. Toutefois, la construction de telles ontologies nécessite beaucoup d'efforts et de temps, d'où une préoccupation d'outiller les créateurs.
La deuxième conférence thématique a été présentée et animée par France Henri, professeure et chercheure au Centre LICEF de TÉLUQ/UQAM, et Bernadette Charlier, professeure à l'Université de Fribourg (Suisse). Elles ont rappelé l'historique de la pratique de la scénarisation en éducation, de son origine dans le domaine de l'audiovisuel jusqu'à aujourd'hui. Elles ont soulevé des questions comme : peuton normaliser la conception d'un scénario pédagogique qui suppose une originalité d'action, un souci particulier d'implication de l'apprenant afin qu'il développe une démarche active et des attitudes autonomes? Selon Henri et Charlier, quelles que soient les qualités d'un scénario pédagogique et celles d'un étudiant, ce qui importe vraiment, c'est la possibilité de les ajuster au moment de leur exploitation dans les situations d'apprentissage.

Aux conférences se sont ajoutées deux tables rondes, en lien avec les aspects de la problématique abordée par les orateurs invités. La première portait sur la modélisation des scénarios pédagogiques et des connaissances ainsi que sur la nécessité de distinguer ces deux activités de modélisation. On y a conclu que le scénario était le lieu d'orchestration de tous les autres modèles (modèle de l'apprenant, modèle de collaboration, modèle des connaissances). Celui qui s'intéresse à la modélisation de l'apprenant ne s'y prendra pas de la même façon que celui qui veut modéliser des connaissances. Mais alors pourquoi modéliser? Plusieurs réponses peuvent être fournies à cette question : pour analyser une situation, pour mieux comprendre un problème ou un domaine, pour mieux communiquer entre membres d'équipes pluridisciplinaires, etc. Tous ces objectifs sont recevables. En fait, les activités de modélisation varient selon le point de vue de celui qui modélise. On peut également modéliser pour représenter des connaissances d'un domaine et pouvoir les réutiliser, les partager. À ce propos, la construction d'une ontologie peut devenir une activité d'apprentissage significative si elle est complétée par l'apprenant. Il y a une surutilisation du terme « ontologie » et on rêve encore de l'ontologie universelle. Par contre, il a été montré à diverses reprises que cela n'était pas possible et, en général, les membres de la communauté disent construire des ontologies pour des besoins particuliers. 
La deuxième table ronde portait sur deux questions. La première s'interrogeait sur l'avantage à normaliser la conception de scénarios pédagogiques, tandis que la deuxième visait à déterminer le regard des acteurs éducatifs sur l'utilisation des scénarios (conception, réutilisation, mutualisation) ainsi que sur le développement d'outils, de langages de modélisation ou d'éditeurs de scénarios. Les réflexions se sont orientées vers des questionnements visant à distinguer normalisation et formalisation, à identifier les destinataires de la scénarisation (les parents, le personnel, les étudiants, par exemple), à argumenter sur le choix d'approches descendantes ou ascendantes et à tenter d'en mesurer les effets.

Le colloque s'est terminé par une synthèse au cours de laquelle il a été question, entre autres, de design participatif, de la prise en compte nécessaire des besoins de l'usager, de l'importance du dialogue entre la pédagogie et la technologie et de l'engagement essentiel des institutions dans l'implantation de changements. Certains peuvent se sentir envahis par une pléthore d'objets d'apprentissage qu'ils considèrent pour l'instant comme non adaptés aux besoins de la pédagogie. Mais, comme cela fut rappelé, « les normes n'ont peut-être pas leur place dans le quotidien du pédagogue pour l'instant, mais elles sont bien là et il ne faut pas faire comme si elles n'existaient pas. Il faut réfléchir à ce que les normes peuvent nous apporter et à ce que nous pouvons apporter aux normes ». Les rôles de l'apprenant et des enseignants changent et il faut prendre le temps de s'adapter à de tels changements.

En résumé, ce second colloque a démontré la nécessité de renforcer la thématique de recherche et de l'articuler davantage avec les besoins institutionnels et sociaux représentés par l'intégration raisonnée des technologies numériques dans les systèmes éducatifs. À la suite de cette manifestation, le comité scientifique a décidé, d'une part, de publier les actes sous format numérique et, d'autre part, d'éditer ce numéro spécial, Scénariser l'apprentissage, une activité de modélisation, dans la Revue internationale des technologies en pédagogie universitaire qui, comme précisé sur le site web de la revue (http://www.profetic.org/revue/) « est consacrée à la diffusion, en accès libre, d'expériences, de pratiques, d'évaluations, de réflexions critiques et de recherches sur l'intégration des TIC en enseignement supérieur ».

\section{Le contenu du numéro spécial}

Le numéro spécial Scénariser l'apprentissage, une activité de modélisation se compose de six articles répartis en deux catégories : Scénariser, un acte pédagogique et Scénariser une activité de formalisation. Chacune des parties est introduite par un texte de base sur la problématique abordée et suivie de deux textes venant l'illustrer dans la pratique.

Pour construire ce numéro spécial, nous avons repris l'organisation du colloque 2007 Scénariser les activités de l'apprenant: une activité de modélisation qui s'était imposée à la lecture des papiers sélectionnés. La première partie du colloque, Formalisation du scénario, a été fortement orientée vers l'instrumentation du processus de scénarisation conduisant à une formalisation de 1'activité pour une implémentation informatique. La deuxième partie, Scénarisation pédagogique, s'est quant à elle davantage orientée vers des questionnements liés à la problématique de l'intégration de la scénarisation pédagogique dans les pratiques éducatives.

Il nous a semblé naturel d'ouvrir ce numéro spécial avec une mise en perspective historique du concept de scénarisation pédagogique en éducation pour aborder ensuite la complexité du processus que ce concept recouvre. En particulier, l'instrumentation cognitive et technique de ce processus devient essentielle dans un contexte où les technologies de l'information ne cessent d'évoluer, et où les produits de cette évolution ne cessent de s'imposer aux enseignants et aux apprenants.

\section{Première partie : Scénariser, un acte pédagogique}

Cette première partie du numéro spécial est introduite par le texte d'Henri, Compte et Charlier La scénarisation pédagogique dans tous ses débats..., une contribution à saveur épistémologique qui tente de définir ce qu'est la scénarisation. Les auteures se préoccupent de la dimension « connaissance du domaine » et disent comment le concept 
de scénarisation a pris forme dans le temps, et en sciences de l'éducation. Elles partent d'une définition originelle du concept de scénarisation pour mieux comprendre l'évolution qu'il a subie. Ce faisant, elles abordent certains éléments de problématique de la scénarisation selon trois perspectives : les potentialités de la scénarisation médiatique, l'intégration de la scénarisation dans la pratique pédagogique et l'énaction (perception-action) (Varela, 1989) du scénario par l'apprenant.

Attaché à cette problématique de la scénarisation pédagogique dans la pratique éducative, le texte d'Emin et al., Stratégies d'élaboration, de partage et de réutilisation de scénarios pédagogiques, propose une analyse des activités de scénarisation menées par deux enseignants experts s'inscrivant dans une démarche de recherche en didactique des sciences. Comme l'indiquent les auteurs, ces enseignants ont élaboré et testé plusieurs versions de scénarios mettant en œuvre une démarche d'investigation et précisant l'organisation des activités à réaliser par les élèves et les enseignants. À partir de l'analyse effectuée, les auteurs tentent de dégager un modèle qui intègre les dimensions didactique, pédagogique ou situationnelle dans l'élaboration des scénarios, et proposent quelques pistes pour le partage et la réutilisation de ces scénarios.

Le troisième article de cette première partie décrit un processus d'ingénierie éducative orienté compétences d'une activité d'autoévaluation et son intégration à un scénario pédagogique de cours à distance. Cette description est documentée par deux cas d'application dans un contexte de formation universitaire à distance. Le travail d'ingénierie utilise une approche en autogestion des compétences pour concevoir l'activité d'autoévaluation et l'instrumentaliser avec l'outil logiciel web d'autodiagnostic des compétences, nommé Compétences+. Cet acte d'ingénierie pédagogique porte l'attention de l'enseignant-concepteur sur une démarche centrée sur l'apprentissage dans une perspective d'autogestion des connaissances et de sa validation dans un usage en contexte isomorphe à une situation professionnelle réelle.

\section{Deuxième partie :}

\section{Scénariser, une activité de formalisation}

La deuxième partie de ce numéro spécial porte sur l'expression des scénarios pédagogiques sous forme d'objets d'apprentissage, à l'aide d'un format qui les rend plus facilement portables et réutilisables. Elle est introduite par une contribution de Paquette dans laquelle la scénarisation pédagogique est définie comme un processus complexe dont l'instrumentation devient essentielle. L'auteur présente une instrumentation du processus de scénarisation composée de divers outils graphiques de modélisation, d'un processus de standardisation des scénarios comme une démarche instrumentale pour favoriser la réutilisation et l'adaptation des approches pédagogiques, et l'intégration des scénarios sous forme $d^{\prime}$ " objets d'apprentissage », dans un référentiel de ressources-scénarios réutilisables pour construire d'autres scénarios. En dernier lieu, l'auteur introduit les scénarios de conception comme des outils méthodologiques à l'usage des ingénieurs pédagogiques pour les aider à améliorer la qualité des scénarios d'apprentissage.

La contribution de David et al. examine la notion de modélisation pédagogique, la définit comme une description formelle d'un scénario, de manière à pouvoir l'opérationnaliser sur une plateforme technologique. La question de la scénarisation des SACI (situations d'apprentissage collectives instrumentées) s'inscrit dans une démarche de réutilisation et de mutualisation non seulement des scénarios pédagogiques, mais surtout « de pratiques rendues possibles par l'usage de plateformes de téléenseignement ». Les auteurs proposent un formalisme d'expression des scénarios de SACI qui veut permettre une réutilisation par les acteurs du dispositif de la plateforme technologique.

Enfin, Nodenot, dans son article Scénarisation pédagogique et modèles conceptuels d'un EIAH : que peuvent apporter les langages visuels? propose d'étudier les apports des langages visuels pour la scénarisation pédagogique d'un environnement informatique pour l'apprentissage humain (EIAH). Il examine les modèles conceptuels que ces langages permettent de produire à partir de l'évaluation de différents langa- 
ges visuels. Plusieurs apports sont soulignés et discutés. Les langages visuels sont des outils exploratoires qui aident les concepteurs à définir les contours d'un EIAH; ces langages permettent aussi aux différents acteurs d'échanger des points de vue au sein d'une équipe de développement pour aboutir à une solution viable. Au-delà de leurs aspects graphiques, l'expressivité des langages visuels devrait s'appuyer sur trois piliers essentiels : des primitives de conception tangibles pour les acteurs impliqués dans l'équipe de développement, une capacité à exprimer différentes perspectives d'un EIAH et différents niveaux d'abstraction pour ces perspectives.

\section{Conclusion}

Les deux colloques SCÉNARIO et leurs actes publiés, ainsi que ce numéro spécial, témoignent de toute la richesse de cette thématique. L'un des objectifs de ces événements scientifiques était de permettre aux praticiens et aux chercheurs de différentes disciplines et de différentes cultures préoccupés par la problématique de la scénarisation des activités d'apprentissage de partager leurs questionnements, l'avancement de leurs travaux ainsi que leurs productions. Nous espérons que ce numéro spécial contribuera largement à cet objectif en mettant en exergue les connaissances nouvelles issues de ces travaux et en offrant la possibilité de les réinvestir dans l'amélioration des environnements numériques d'apprentissage et des pratiques éducatives.

\section{Références}

Centre de recherche LICEF (dir.). (2007). Scénariser les activités de l'apprenant : une activité de modélisation. Actes du colloque Scénario 2007. Récupéré le 3 mars 2008 du site LICEF, http: / / www. licef.teluq.uquebec.ca

Koper, R. et Tattersall, C. (dir.). (2005). Learning design. A handbook on modelling and delivering networked education and training. Berling Heidelberg : Springer-Verlag.

Paquette, G. (2006). Introduction à la spécification IML-LD d'une perspective d'ingénierie pédagogique. Récupéré le 7 septembre 2007 du site IDLD (Déploiement et implantation de la spécification pour les designs d'apprentissage), http://www.idld.org/ Methodology/tabid/174/Default.aspx

Pernin, J.-P. et Godinet, H. (2006). Scénariser l'enseignement et l'apprentissage : une nouvelle compétence pour le praticien? Actes du colloque Scénario 2006. Récupéré le 3 mars 2008 du site http:/ / www.inrp.fr/ publications / edition-electronique/

Savard, E. (2007). Scénario 2007, le 2e colloque international sur les scénarios pédagogiques. Récupéré le 3 mars 2008 du site PROFETIC, http:/ / www.profetic.org/spip.php?article8907

Varela, F. J. (1989). Connaître les sciences cognitives. Tendances et perspectives. Paris : Seuil. 